\title{
The Effect of Learning Feedback Delay on the Growth of E-commerce Web Sites
}

\author{
Xiao-ji Wan ${ }^{1}$ Gui-shi Deng ${ }^{1}$ Yang Bai $^{1,2}$ Zhi-guo $\mathrm{Zhu}^{3}$ \\ ${ }^{1}$ Institute of Systems Engineering, Dalian University of Technology, Dalian 116024, \\ P.R.China.Email:wan-2002-xiaoji@163.com,denggs@dlut.edu.cn \\ ${ }^{2}$ College of Information Technology, Eastern Liaoning University, Dandong 118003 , \\ P.R.China.Email:by1997@163.com \\ ${ }^{3}$ Kingbridge Business School, Dongbei University of Finance and Economics, Dalian \\ 116023, P.R.China.Email: 82119022@qq.com
}

\begin{abstract}
The learning feedback delay reflecting renewal speed greatly affects the growth of e-commerce web sites. This paper firstly presents a nonlinear competition model ex-plaining the growth mechanism of commercial sites and studies the action principle of initial conformation condition, renewal speed, competitive intensity and user prefe-rence on sites. Secondly, from the dynamics perspective, we discuss the action result of learning feedback delay (time delays) and find the relationship between its critical value and two sites sharing market or periodically fluctuating. Finally, we demonstrate the conclusion by some numerical simulations and provide some basis and refer-ences for website builders.
\end{abstract}

Keywords: e-commerce web sites, learning feedback delay, growth mechanism, competition model, system stability, Hopf bifurcation

\section{Introduction}

With the development of e-commerce, there are increasingly commercial websites emerging one after another. The fact that more and more people and enterprises step in this field leads to fierce competition. The current competition will easily appear in "Coexistence", "Extinction", "Winner-take-all" and etc. Because the competition in the virtual internet setting is more prominent and vivid than in other fields', the internal key mechanisms and causes are worth studying and exploring. There have been some researches in this field recently, involving the positive feedback theory, network effect theory and network marketing theory ${ }^{[1]}$ of the network economics and the web sites' operating strategic intents, income patterns, sale modes and channels, extension modes of the marketing. From the dynamics, M.Maurer and A.Huberman ${ }^{[2]}$ firstly proposed the following e-commerce web sites competition model based on the classical Lotka-volterra competitive equations in biology and studied its internal growth mechanism.

$$
\frac{d x_{i}}{d t}=\alpha_{i} x_{i}\left(\beta_{i}-x_{i}\right)-\sum_{j \neq i, j=1}^{n} \gamma_{i j} x_{i} x_{j} .
$$

Ref.[3] studies the system stability of (1) and gives some corresponding strategies aiming at the different market settings. Ref.[4] extends system (1) to a model which accounts for cooperation and competition at the same time and gives a series of interesting strategies for cooperating with small sites to win. As complement, Ref.[5] gives the complete strategic classification for the model in [4] without 
any hypothesis. Under coefficients are symmetrical, Ref. [6] studies the system stability, the direction and stability of bifurcated periodic solutions for system (1) with a single time delay by using the center manifold theorem and the normal form theory. Ref.[7] qualitatively analyzes system (1) with market segment and shows some conditions on which small websites can survive by using the market segmentation strategy. Based on the model (1), the Refs.[8-10] establish a novel e-commerce web sites competition model (2) in which the characteristic of "better for better" and the effect of "user preference" are considered and the dynamical behaviors and the phenomenon of "Winner-take-all" are studied.

$$
\frac{d x_{i}}{d t}=\alpha_{i} x_{i}\left(\beta_{i}-x_{i}\right)-\sum_{j \neq i, j=1}^{n} \gamma_{i j}\left(1+\omega_{i j}\left(x_{j}-x_{i}\right)\right) x_{i} x_{j}
$$

Some of mentioned references only discuss the competitive system with a single learning feedback delay and don't consider the characteristic of "better for better" of e-commerce sites and the effect of "user preference". In addition, although others refer to these factors, they don't analyze the effect of learning feedback delay reflecting the renewal speed on the growth of sites.

In fact, both the learning feedback delay and user preference can restrict the growth of e-commerce sites. If website builders don't pay more attention to these factors, sites will loss the competitive advantages. In order to make up these shortages in literatures, the paper will constitute a novel e-commerce website competition model and study the influence of these factors on the growth of sites.

The remainder of the letters is organized as follows. In Section 2, a nonlinear website competition model is established. In Section 3, the effect of learning feed- back delay on the growth of e-commerce websites is studied. Finally, some numerical simulations are performed to illustrate the analytical results.

\section{Modeling}

In the real world, the users who go shopping online usually tend to consume at some websites with high credit, complete goods and beautiful interface. These sites will become stronger and obtain more profits than others. That is, e-commerce web sites have the characteristic of "better for better". Moreover, sites of mutual competition will know themselves as well as their opponents in order to keep predominance. Once realized their opponents selling products with lower price and constantly renewing products or aligning with other sellers, other sites will capture internal and external information by combining own advantages and sources and adopt some strategies to deal with. At this time, the learning feedback delay will turn up. Obviously, the less time the learning feedback delay wastes, the more advantages sites will have.

Generally, the growth speed of site $i$ is proportional to its natural growth rate and reversely proportional to the competitive impact from opponents. On the other hand, user preferences and learning feedback delays will also limit sites development. In order to objectively reflect the competition among e-commerce sites, we establish the following competition model with time delays:

$$
\begin{aligned}
& \frac{d x_{i}(t)}{d t}=\alpha_{i} x_{i}(t)\left(\beta_{i}-x_{i}\left(t-\tau_{i}\right)\right)- \\
& \sum_{j \neq i, j=1}^{n} \gamma_{i j}\left(1+\omega_{i j}\left(x_{j}(t)-x_{i}(t)\right)\right) x_{i}(t) x_{j}(t),
\end{aligned}
$$

where ${ }^{x_{i}}$ is the fraction of the population that is a customer of site i. $\alpha_{i}$ is the growth 
rate of site $\mathrm{i}$ without any competitions and $^{\beta_{i}}$ denotes their capacity to service a fraction of the customers. When other sites offer competing services, ${ }^{x_{i} x_{j}}$ denotes the fraction of using both sites at the same time. Because both sites provide similar services, some customers will stop using one or the other. ${ }^{\gamma_{i j} x_{i} x_{j}}$ stands for the rate customers stop using site $\mathrm{i}$ and visit site $\mathrm{j}$ because of site $\mathrm{j} \cdot \gamma_{i j}$ is competitive strength between site $\mathrm{i}$ and $\mathrm{j}$. In addition, because of the characteristic of "better for better" of e-commerce web sites, customers usually "preferentially" use the services provided by sites with more customers, here ${ }^{\omega_{i j}\left(x_{j}-x_{i}\right)}$ is the effect of preferential choice, and ${ }^{\omega_{i j}}$ expresses effect strength. ${ }_{i j}\left(1+\omega_{i j}\left(x_{j}-x_{i}\right)\right) x_{i} x_{j}$ denotes the effect of mutual users' preference between sites $\mathrm{i}$ and $\mathrm{j}$ on Churn.

$\tau^{\tau_{i}}$ represents the required time that site $\mathrm{i}$ collects internal and external information and carries out strategies. $0 \leq x_{i} \leq 1, \alpha_{i}>0$, $0<\beta_{i} \leq 1,0<\omega_{i j}<\min \left\{1, \gamma_{i j}\right\}, \tau_{i}>0, i, j=1,2, \ldots, n$.

Because the competition of multi-sites are very complex, here we only consider two e-commerce web sites and set each corresponding coefficient to be equal. Namely, we consider the following model

$$
\left\{\begin{array}{l}
\frac{d x_{1}(t)}{d t}=\alpha x_{1}(t)\left(\beta-x_{1}(t-\tau)\right)-\gamma\left(1+\omega\left(x_{2}(t)-x_{1}(t)\right)\right) x_{1}(t) x_{2}(t), \\
\frac{d x_{2}(t)}{d t}=\alpha x_{2}(t)\left(\beta-x_{2}(t-\tau)\right)-\gamma\left(1+\omega\left(x_{1}(t)-x_{2}(t)\right)\right) x_{1}(t) x_{2}(t) .
\end{array}\right.
$$

In what follows, we will study the effect of initial conformation condition, renewal speed, competitive intensity and user preference on the growth of two ecommerce web sites.

\section{Model analyses}

\subsection{Without learning feedback delay}

Obviously, when system (4) doesn't consider the learning feedback delay reflecting renewal speed, namely $\tau=0$, it exists the following steady states:

$P_{0}(0,0), P_{1}(\beta, 0), P_{2}(0, \beta), P_{3}\left(\frac{\alpha \beta}{\alpha+\gamma}, \frac{\alpha \beta}{\alpha+\gamma}\right), P_{4}(u, v), P_{5}(v, u)$,

where

$$
u=\frac{\alpha-\gamma+\sqrt{-\alpha^{2}+\gamma^{2}+2 \alpha \beta \gamma \omega}}{2 \gamma \omega},
$$

$v=\frac{\alpha-\gamma-\sqrt{-\alpha^{2}+\gamma^{2}+2 \alpha \beta \gamma \omega}}{2 \gamma \omega}$.

By straightforward computation, when $\frac{\alpha}{\beta \omega+\sqrt{1+\beta^{2} \omega^{2}}} \leq \gamma \leq \frac{\alpha}{1+\beta \omega}$,

the equilibriums ${ }_{P_{4}, P_{5}}$ will be meaningfulness ( $u, v$ are non-negative).

For the stability of the above equilibriums, Ref.[8] did some investigations and obtained the following conclusion.

Lemma 1. When both two e-commerce websites don't consider the learning feedback delay, namely $\tau=0$ in system (4), the stability of the upper equilibriums are

(1)For all $\gamma>0$, the equilibrium ${ }^{P_{0}(0,0)}$ is unstable;

(2)when $\gamma>\frac{\alpha}{1+\beta \omega} \quad$, the equilibriums $P_{1}(\beta, 0), P_{2}(0, \beta)$ are stable and $P_{3}\left(\frac{\alpha \beta}{\alpha+\gamma}, \frac{\alpha \beta}{\alpha+\gamma}\right)$ is unstable;

(3)when $\frac{\alpha}{\beta \omega+\sqrt{1+\beta^{2} \omega^{2}}}<\gamma<\frac{\alpha}{1+\beta \omega}$, the equilibriums

$P_{1}(\beta, 0), P_{2}(0, \beta), P_{3}\left(\frac{\alpha \beta}{\alpha+\gamma}, \frac{\alpha \beta}{\alpha+\gamma}\right)$ are unstable, $P_{4}(u, v), P_{5}(v, u)$ exist and are stable;

(4) when $0<\gamma<\frac{\alpha}{\beta \omega+\sqrt{1+\beta^{2} \omega^{2}}}$, the equilibriums $P_{1}(\beta, 0), P_{2}(0, \beta)$ are unstable and $P_{3}\left(\frac{\alpha \beta}{\alpha+\gamma}, \frac{\alpha \beta}{\alpha+\gamma}\right)$ is stable.

According to the lemma 1 , if ecommerce sites competing with each other don't consider the learning feedback representing the update speed, no matter 
what the competition is strong $(\gamma>\alpha)$ or weak $(\gamma<\alpha)$, the phenomenon that both sites tend to be extinct is impossible; If the competition strength lies in strong or second weak, one site may monopolize market and result in "Winner-take-all". Which site can occupy the whole market is up to the initial market share, one with higher market share will monopolize market and the rest will tend to extinction. In addition, the case that two webs sharing market have the same share is impossible; When the competition strength drops to $\frac{\alpha}{\beta \omega+\sqrt{1+\beta^{2} \omega^{2}}}<\gamma<\frac{\alpha}{1+\beta \omega}$, these situations that the single site monopolizes market and two sites with the same share enjoy market are impossible. But two sites with different shares may occupy market at the same time. Here, the site with higher initial market share will ac- quire more advantages; If the competition intensity of sites decreases to $\quad 0<\gamma<\frac{\alpha}{\beta \omega+\sqrt{1+\beta^{2} \omega^{2}}}$, then neither of sites monopolizes market but two sites with the same share will occupy the whole market.

\subsection{With learning feedback delay}

In this part, we will mainly discuss the effect of the learning feedback delay reflecting the update speed on the growth of two e-commerce web sites.

Obviously, the points $P_{i}\left(x_{1}^{*}, x_{2}^{*}\right), i=0,1, \ldots, 5$ are also the singular points of system (4). Using the following transformation for $P_{i}\left(x_{1}^{*}, x_{2}^{*}\right)$,

$u_{1}(t)=x_{1}(t)-x_{1}^{*}, u_{2}(t)=x_{2}(t)-x_{2}^{*}$,

then the system (4) will convert to

$$
\left\{\begin{array}{l}
\frac{d u_{1}(t)}{d t}=\left[\alpha\left(\beta-x_{1}^{*}\right)-\gamma x_{2}^{*}\left(1+\omega\left(x_{2}^{*}-2 x_{1}^{*}\right)\right)\right] u_{1}(t)-\alpha x_{1}^{*} u_{1}(t-\tau)-\gamma x_{1}^{*}\left[1+\omega\left(2 x_{2}^{*}-x_{1}^{*}\right)\right] u_{2}(t) \\
-\alpha u_{1}(t) u_{1}(t-\tau)-\gamma\left[1+2 \omega\left(x_{2}^{*}-x_{1}^{*}\right)\right] u_{1}(t) u_{2}(t)+\gamma \omega x_{2}^{*}\left(u_{1}(t)\right)^{2}-\gamma \omega x_{1}^{*}\left(u_{2}(t)\right)^{2}-\gamma \omega u_{1}(t) u_{2}(t)\left[u_{2}(t)-u_{1}(t)\right], \\
\frac{d x_{2}(t)}{d t}=-\gamma x_{2}^{*}\left[1+\omega\left(2 x_{1}^{*}-x_{2}^{*}\right)\right] u_{1}(t)+\left[\alpha\left(\beta-x_{2}^{*}\right)-\gamma x_{1}^{*}\left(1+\omega\left(x_{1}^{*}-2 x_{2}^{*}\right)\right)\right] u_{2}(t)-\alpha x_{2}^{*} u_{2}(t-\tau) \\
-\alpha u_{2}(t) u_{2}(t-\tau)-\gamma\left[1+2 \omega\left(x_{1}^{*}-x_{2}^{*}\right)\right] u_{1}(t) u_{2}(t)-\gamma \omega x_{2}^{*}\left(u_{1}(t)\right)^{2}+\gamma \omega x_{1}^{*}\left(u_{2}(t)\right)^{2}+\gamma \omega u_{1}(t) u_{2}(t)\left[u_{2}(t)-u_{1}(t)\right],
\end{array}\right.
$$

The linearization of (5) at is

$$
\left\{\begin{array}{l}
\frac{d u_{1}(t)}{d t}=\left[\alpha\left(\beta-x_{1}^{*}\right)-\gamma x_{2}^{*}\left(1+\omega\left(x_{2}^{*}-2 x_{1}^{*}\right)\right)\right] u_{1}(t)-\alpha x_{1}^{*} u_{1}(t-\tau)-\gamma x_{1}^{*}\left[1+\omega\left(2 x_{2}^{*}-x_{1}^{*}\right)\right] u_{2}(t), \\
\frac{d x_{2}(t)}{d t}=-\gamma x_{2}^{*}\left[1+\omega\left(2 x_{1}^{*}-x_{2}^{*}\right)\right] u_{1}(t)+\left[\alpha\left(\beta-x_{2}^{*}\right)-\gamma x_{1}^{*}\left(1+\omega\left(x_{1}^{*}-2 x_{2}^{*}\right)\right)\right] u_{2}(t)-\alpha x_{2}^{*} u_{2}(t-\tau),
\end{array}\right.
$$
is

Thus, the characteristic equation of (6)

$$
\lambda^{2}+a_{0} \lambda+b_{0}+\left(a_{1} \lambda+b_{1}\right) e^{-\lambda \tau}+c_{0} e^{-2 \lambda \tau}=0,
$$

where

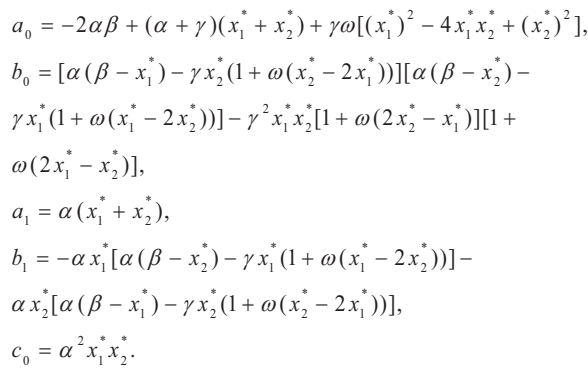


Without loss of generality, we only discuss the effect of the learning feedback delay on the equilibrium $P_{3}\left(\frac{\alpha \beta}{\alpha+\gamma}, \frac{\alpha \beta}{\alpha+\gamma}\right)$ in which both two ecommerce sites have the same market share in the stable state.

By calculation, the characteristic equation (7) can be described by

$$
\chi_{1}(\lambda) \chi_{2}(\lambda)=0
$$

where $\quad \chi_{1}(\lambda)=\lambda+M_{1}+N_{1} e^{-\lambda \tau}, \chi_{2}(\lambda)=\lambda+M_{2}+N_{2} e^{-\lambda \tau}$, $M_{1}=\frac{\alpha \beta \gamma}{\alpha+\gamma}, M_{2}=-\frac{\alpha \beta \gamma}{\alpha+\gamma}-2 \gamma \omega\left(\frac{\alpha \beta}{\alpha+\gamma}\right)^{2}, N_{1}=\frac{\alpha^{2} \beta}{\alpha+\gamma}, N_{2}=\frac{\alpha^{2} \beta}{\alpha+\gamma}$

In order to investigate the distribution of the roots of Eq.(7), we need introduce the following lemma[11].

Lemma 2. Consider the exponential polynomial

$$
\begin{aligned}
& P\left(\lambda, e^{-\lambda \tau_{1}}, \ldots, e^{-\lambda \tau_{m}}\right) \\
& =\lambda^{n}+p_{1}^{(0)} \lambda^{n-1}+\ldots+p_{n-1}^{(0)} \lambda+p_{n}^{(0)} \\
& =\left[p_{1}^{(1)} \lambda^{n-1}+\ldots+p_{n-1}^{(1)} \lambda+p_{n}^{(1)}\right] e^{-\lambda \tau_{1}}+\ldots \\
& +\left[p_{1}^{(m)} \lambda^{n-1}+\ldots+p_{n-1}^{(m)} \lambda+p_{n}^{(m)}\right] e^{-\lambda \tau_{m}},
\end{aligned}
$$

where $_{\tau_{i}} \geq 0$ and $p_{j}^{(i)}, i=1,2, \ldots, m, j=1,2, \ldots, n$ are constants. As $\left(\tau_{1}, \tau_{2}, \ldots, \tau_{m}\right)$ vary, the sum of the order of the zeros of $P\left(\lambda, e^{-\lambda \tau_{1}}, \ldots, e^{-\lambda \tau_{m}}\right)$ on the open right half plane can change if and only if there are zeros crossing the imaginary axis.

Suppose that $i \delta(\delta>0)$ is a root of Eq.(8) in the imaginary axis. Substituting it to the Eq.(8) and separating the real and imaginary parts, we have

$$
\left\{\begin{array}{c}
M_{1}+N_{1} \cos \delta \tau=0 \\
\delta-N_{1} \sin \delta \tau=0
\end{array}\right.
$$

or

$$
\left\{\begin{array}{c}
M_{2}+N_{2} \cos \delta \tau=0, \\
\delta-N_{2} \sin \delta \tau=0,
\end{array}\right.
$$

Obviously, system (9) is equivalent to

$$
\delta^{2}=N_{1}^{2}-M_{1}^{2} .
$$

Therefore, when $\left|N_{1}\right|>\left|M_{1}\right|$, namely $\gamma<\alpha$, Eq.(11) exists a positive root:

$$
\delta=\delta_{1}=\sqrt{N_{1}^{2}-M_{1}^{2}}=\frac{\alpha \beta}{\alpha+\gamma} \sqrt{\alpha^{2}-\gamma^{2}} ;
$$

Likewise, from (10), we can obtain

$$
\delta^{2}=N_{2}^{2}-M_{2}^{2},
$$

then when $\quad\left|N_{2}\right|>\left|M_{2}\right|$, that is $0<\gamma<\frac{\alpha}{\beta \omega+\sqrt{1+\beta^{2} \omega^{2}}}$, Eq.(13) exists a positive root

$\delta=\delta_{2}=\sqrt{N_{2}^{2}-M_{2}^{2}}=\frac{\alpha \beta}{\alpha+\gamma} \sqrt{\alpha^{2}-\gamma^{2}\left(1+\frac{2 \alpha \beta \omega}{\alpha+\gamma}\right)^{2}} ;$

Based on the above discussions, we can get the following lemma.

Lemma 3. For Eq.(11) and Eq.(13),we have

(1)When ${ }^{\gamma \geq \alpha}$, both Eq.(11) and Eq.(13) have no positive roots;

(2) When $\frac{\alpha}{\beta \omega+\sqrt{1+\beta^{2} \omega^{2}}} \leq \gamma<\alpha$, Eq.(11) has one positive $\operatorname{root}^{\delta_{1}}$ and Eq.(13) has no positive root;

(3)When $\quad 0<\gamma<\frac{\alpha}{\beta \omega+\sqrt{1+\beta^{2} \omega^{2}}}$, Eq.(11) and (13) have the positive roots $\delta_{1}$ and $\delta_{2}$, respectively.

Without loss of generality, we suppose that Eq.(11) and (13) exist positive

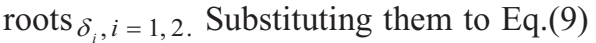
and (10), we can acquire

$$
\begin{aligned}
\tau_{1}^{(\mathrm{j})} & =\frac{1}{\delta_{1}}\left\{\arccos \left(-\frac{M_{1}}{N_{1}}\right)+2 j \pi\right\} \\
& =\frac{\alpha+\gamma}{\alpha \beta \sqrt{\alpha^{2}-\gamma^{2}}}\left\{\arccos \left(-\frac{\gamma}{\alpha}\right)+2 j \pi\right\}, j=0,1,2, \ldots .
\end{aligned}
$$




$$
\begin{aligned}
\tau_{2}^{(j)} & =\frac{1}{\delta_{2}}\left\{\arccos \left(-\frac{M_{2}}{N_{2}}\right)+2 j \pi\right\} \\
& =\frac{\alpha+\gamma}{\alpha \beta \sqrt{\alpha^{2}-\gamma^{2}\left(1+\frac{2 \alpha \beta \omega}{\alpha+\gamma}\right)^{2}}}\left\{\arccos \left(\frac{\gamma}{\alpha}\left(1+\frac{2 \alpha \beta \omega}{\alpha+\gamma}\right)\right)\right. \\
& +2 j \pi\}, j=0,1,2, \ldots
\end{aligned}
$$

Define

$$
\tau_{0}=\min \left\{\tau_{1}^{(0)}, \tau_{2}^{(0)}\right\}
$$

Here, when $\tau_{0}=\tau_{i}^{(0)}, \delta=\delta_{i}, i=1,2$.

Notice that when $\tau=0$, the characteristic Eq.(7) can transform to

$$
\lambda^{2}+\left(a_{0}+a_{1}\right) \lambda+b_{0}+b_{1}+c_{0}=0 .
$$

From the lemma 1, if the following condition is satisfied

$$
(H): 0<\gamma<\frac{\alpha}{\beta \omega+\sqrt{1+\beta^{2} \omega^{2}}},
$$

then all roots of Eq.(18) have positive real parts. In this case, the equilibrium $P_{3}\left(\frac{\alpha \beta}{\alpha+\gamma}, \frac{\alpha \beta}{\alpha+\gamma}\right)$ of system (4) is stable.

On the basis of the lemma 2 and 3, we have

Lemma 4. For the second degree transcendental Eq.(7),

(1)If $\gamma \geq \alpha$, then the positive roots of Eq.(7) and Eq.(18) have the same amount for $\forall \tau \geq 0$;

(2) If

$$
\frac{\alpha}{\beta \omega+\sqrt{1+\beta^{2} \omega^{2}}} \leq \gamma<\alpha, \text { then }
$$

$\tau_{2}^{(0)}$ doesn't exist, $\tau_{0}=\tau_{1}^{(0)}$ and the positive roots of Eq.(7) and Eq.(18) have the same amount for $\tau \in\left[0, \tau_{0}\right)$;
(3) If $0<\gamma<\frac{\alpha}{\beta \omega+\sqrt{1+\beta^{2} \omega^{2}}}$, then the positive roots of Eq.(7) and Eq.(18) have the same amount for $\tau \in\left[0, \tau_{0}\right)$.

Let $\lambda(\tau)=\sigma(\tau)+i \delta(\tau)$ be the root of Eq.(7) near

where

$\sigma\left(\tau_{k}^{(j)}\right)=0, \delta\left(\tau_{k}^{(j)}\right)=\delta_{k}, k=1,2, j=0,1,2 \ldots$ Sub stituting it into the Eq.(7) and taking the derivative with respect to $\tau$, we obtain

$\left[\frac{d \lambda}{d \tau}\right]^{-1}=\frac{\left(2 \lambda+a_{0}\right) e^{2 \lambda \tau}+\left[a_{1}-\tau\left(a_{1} \lambda+b_{1}\right)\right] e^{\lambda \tau}-2 c_{0} \tau}{\lambda\left[\left(a_{1} \lambda+b_{1}\right) e^{\lambda \tau}+2 c_{0}\right]}$,

When $\tau=\tau_{k}^{(j)}$, it is easy to compute the transversality condition $\left[\frac{d \operatorname{Re}(\lambda)}{d \tau}\right]_{\tau=\tau_{k}^{(j)}}^{-1}=\frac{M}{N}$, where

$$
\begin{aligned}
& M=-\delta_{k}\left[a_{1} \delta_{k} \cos \left(\delta_{k} \tau_{k}^{(j)}\right)+b_{1} \sin \left(\delta_{k} \tau_{k}^{(j)}\right)\right]\left[a_{0} \cos \left(2 \delta_{k} \tau_{k}^{(j)}\right)\right. \\
& -2 \delta_{k} \sin \left(2 \delta_{k} \tau_{k}^{(j)}\right)+\left(a_{1}-b_{1} \tau_{k}^{(j)}\right) \cos \left(\delta_{k} \tau_{k}^{(j)}\right)-2 c_{0} \tau_{k}^{(j)}+ \\
& \left.a_{1} \delta_{k} \tau_{k}^{(j)} \sin \left(\delta_{k} \tau_{k}^{(j)}\right)\right]+\delta_{k}\left[2 c_{0}-a_{1} \delta_{k} \sin \left(\delta_{k} \tau_{k}^{(j)}\right)+\right. \\
& \left.b_{1} \cos \left(\delta_{k} \tau_{k}^{(j)}\right)\right]\left[a_{0} \sin \left(2 \delta_{k} \tau_{k}^{(j)}\right)+2 \delta_{k} \cos \left(2 \delta_{k} \tau_{k}^{(j)}\right)\right. \\
& \left.+\left(a_{1}-b_{1} \tau_{k}^{(j)}\right) \sin \left(\delta_{k} \tau_{k}^{(j)}\right)-a_{1} \delta_{k} \tau_{k}^{(j)} \cos \left(\delta_{k} \tau_{k}^{(j)}\right)\right],
\end{aligned}
$$

$N=\delta_{k}^{2}\left[\left(a_{1} \delta_{k} \cos \left(\delta_{k} \tau_{k}^{(j)}\right)+b_{1} \sin \left(\delta_{k} \tau_{k}^{(j)}\right)\right)^{2}+\left(2 c_{0}-a_{1} \delta_{k} \sin \left(\delta_{k} \tau_{k}^{(j)}\right)+b_{1} \cos \left(\delta_{k} \tau_{k}^{(j)}\right)\right)^{2}\right]$.

According to the Hopf bifurcation theory and the above discussion, when $(H): 0<\gamma<\frac{\alpha}{\beta \omega+\sqrt{1+\beta^{2} \omega^{2}}}$ holds and the initial market shares of two sites are unequal, we can obtain the following theorem:

Theorem 1.Let $\tau_{k}^{(j)}, \tau_{0}$ be defined by (1517), then

(1)For $\tau \in\left[0, \tau_{0}\right)$, then all roots of Eq.(7) have negative real parts. In this case, the equilibrium $P_{3}\left(\frac{\alpha \beta}{\alpha+\gamma}, \frac{\alpha \beta}{\alpha+\gamma}\right)$ of system (4) is locally asymptotically stable;

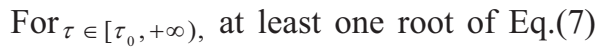
has positive real part, then the equili- 
brium $P_{3}\left(\frac{\alpha \beta}{\alpha+\gamma}, \frac{\alpha \beta}{\alpha+\gamma}\right)$ of system (4) is unstable;

(2)If (1) holds, and $\left[\frac{d \operatorname{Re}(\lambda)}{d \tau}\right]_{\tau=\tau_{k}^{(j)}}^{-1} \neq 0$, then when $\tau=\tau_{k}^{(j)}$, the system (4) exhibits Hopf bifurcations near $P_{3}\left(\frac{\alpha \beta}{\alpha+\gamma}, \frac{\alpha \beta}{\alpha+\gamma}\right)$.

In the light of upper theorem, we know that in a case where one e-commerce site competes weakly against the other site $\left(\gamma<\frac{\alpha}{\beta \omega+\sqrt{1+\beta^{2} \omega^{2}}}<\alpha\right)$ and the initial market shares of two sites are inequality, if the learning feedback delay stays within a range $\left(\tau \in\left[0, \tau_{0}\right)\right)$, two commercial sites having equivalent market shares can coexist; otherwise, this kind of coexistence case cannot take place. Instead of this, sites will periodically fluctuate or irregularly growth. Such may result from the differences of updating speed, competitive intensity, users' preference and etc. Therefore, in order to sustain the market stability and obtain the competitive advantage, website builders should enhance the renewal speed of sites by comprehensive arrangement and attract more customers to visit own sites by advertising or improving the services quality of sites.

Next section, we will demonstrate the conclusion by one example.

\section{Numerical simulations}

Assume that there are only two ecommerce websites competing with each other at a certain internet market and their initial market shares are $x_{1}(0)=0.7$ and $x_{2}(0)=0.3$, respectively. If there no any competition, their natural growth rate is $\alpha=0.6$ and saturated rate of increment is $\beta=1$. Consider the factor of user preference, and set its effect strength is $\omega=0.15$. Thus we have the following model:

$\left\{\begin{array}{l}\frac{d x_{1}(t)}{d t}=0.6 x_{1}(t)\left(1-x_{1}(t-\tau)\right)-\gamma\left(1+0.15\left(x_{2}(t)-x_{1}(t)\right)\right) x_{1}(t) x_{2}(t), \\ \frac{d x_{2}(t)}{d t}=0.6 x_{2}(t)\left(1-x_{2}(t-\tau)\right)-\gamma\left(1+0.15\left(x_{1}(t)-x_{2}(t)\right)\right) x_{1}(t) x_{2}(t),\end{array}\right.$

By straightforward computation, when the competitive intensity $0<\gamma<0.5167$, the condition $_{(H)}$ holds. Without loss of generality, we choose $\gamma=0.51$, in this case, the system (21) can be described by

$\left\{\begin{array}{l}\frac{d x_{1}(t)}{d t}=0.6 x_{1}(t)\left(1-x_{1}(t-\tau)\right)-0.51\left(1+0.15\left(x_{2}(t)-x_{1}(t)\right)\right) x_{1}(t) x_{2}(t), \\ \frac{d x_{2}(t)}{d t}=0.6 x_{2}(t)\left(1-x_{2}(t-\tau)\right)-0.51\left(1+0.15\left(x_{1}(t)-x_{2}(t)\right)\right) x_{1}(t) x_{2}(t),\end{array}\right.$

(22)

According to the discussions of Section 3, system (22) has equilibrium $P_{3}(0.5405,0.5405)$, and

$\tau_{1}^{(j)}=15.1408+36.7764 j$ $\tau_{2}^{(j)}=3.0959+124.5961 j, j=0,1,2, \ldots$. Set $\tau_{0}=\tau_{2}^{(0)}=3.0959$, from theorem 1, we know that when $\tau \in[0,3.0959)$, the equilibrium $P_{3}$ is locally asymptotically stable (see Fig.1). And because the transversality condition $\left[\frac{d \operatorname{Re}(\lambda)}{d \tau}\right]_{\tau=\tau_{0}}^{-1}=9.5069 \neq 0$, when $\tau$ crosses through its critical value $\tau_{0}$, system (22) exhibits Hopf bifurcations (see Fig.2). By simulating, we also find that as $\tau$ increases, the system (22) will appear in period doubling bifurcation (see Fig.3) and unstable case (see Fig.4). 

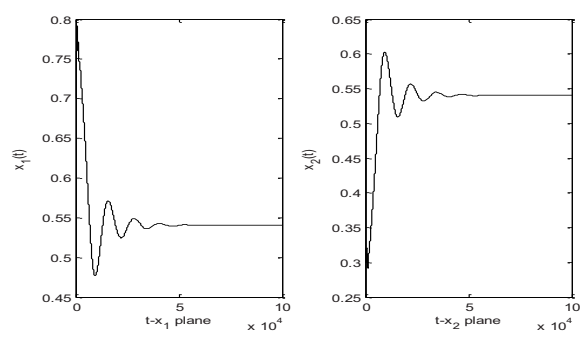

Fig. 1: Competitive intensity $\gamma_{\gamma=0.51}$, the learning feedback delay $\tau=3$, two e-commerce websites with equivalent market share can enjoy the whole market.
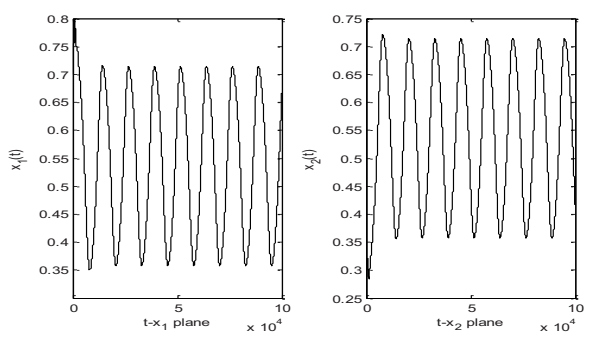

Fig. 2: Competitive intensity $\gamma=0.51$, the learning feedback delay $\tau=3.2$, two e-commerce websites with equivalent market share periodically fluctuate.
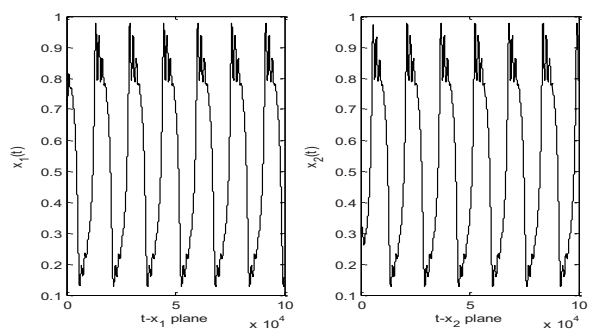

Fig. 3: Competitive intensity $\gamma=0.51$, the learning feedback delay ${ }_{\tau=3.7}$, two e-commerce websites with equivalent market share show period doubling fluctuation.
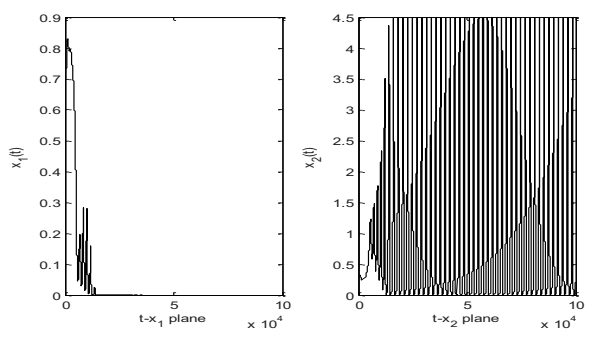

Fig. 4: Competitive intensity $\gamma=0.51$, the learning feedback delay ${ }_{\tau=4.1}$, two e-commerce websites with equivalent market share cannot enjoy the whole market.

\section{Conclusions}

In this paper, we discuss the effect of learning feedback delay reflecting the renewal speed on the growth of commercial sites from dynamical perspective. By setting the competition strength and the learning feedback delay as analysis coefficients, we obtain the conclusions: if two e-commerce sites compete weakly with each other and the learning feedback delay lies in a certain range, then two sites with equivalent share can share the whole market. However, if the learning feedback delay surpasses its threshold, then the market share of sites may periodically fluctuate or irregularly vary. This model objectively reflects the growth mechanism of e-commerce sites and the results found provide basis and references for website builders.

We will proceed in the further study on the development strategies of sites and spread and exploitation of this model.

\section{Acknowledgements}

This work was supported by the National Natural Science Foundation of China (Grant No.70972059, 70890080, 70890083). 


\section{References}

[1] Zhang Jie-yi, "Research on the core competence of online payment enterprises," Beijing: Beijing Jiaotong University, 2008(6). (in Chinese)

[2] Sebastian M. Maurer, Bernardo A. Huberman, "Competitive dynamics of web sites," Journal of Economic Dynamics \& Control, pp.2195-2206, 2003.

[3] Luis Lopez, Miguel A.F. Sanjuan, "Defining strategies to win in the internet market," Physica A, pp.512534, 2001.

[4] Yuanshi Wang, Hong Wu, "Dynamics of a cooperation-competition model for the www market," Physica A, pp.609-620, 2004.

[5] Jifa Jiang, Zhixin Cheng, "The complete strategic classification for a cooperation competition model in the www market," Physica A, pp.527-536, 2006.

[6] Min Xiao, Jinde Cao, "Stability and Hopf bifurcation in a delayed competitive web sites model," Physics Letters A, pp.138-150, 2006.

[7] Yawei Ren, Deli Yang, Xinjun Diao, "Market segmentation strategy in internet market," Physica A, pp.1688 1698, 2010.

[8] Li Yanhui, Zhu Siming, "Analysis of a type of competition model of Ecommerce sites," ACTA SCIENTIARUM NATURALIUM UNIVERSITATIS SUNYATSENI,pp. 610, 2003. (in Chinese)

[9] Li Yanhui, Zhu Siming, "Qualitative analysis of n-dimensional competitive systems," Nonlinear Analysis, pp.700-719, 2006.

[10] Li Yanhui, ZhuSiming, "Competitive dynamics of e-commerce web sites," Applied Mathematical Modelling, pp.912-919, 2007.

[11] Ruan S, Wei J, "On the zeros of transcendental func-tions with applications to stability of delay differential equations with two delays," Dynamics of Continuous, Discrete and Impulsive systems, pp.863-874, 2003. 